\title{
Leucocyte Differential Count and Pregnancy Induced Hypertension: Implication for Risk and Disease Assessment
}

\author{
Nafiu Amidu, ", Benedict Boateng Antuamwine ${ }^{1}$, Martin Awe Akilla ${ }^{1}$, \\ William Kwame Boakye Ansah Owiredu², Otchere Addai-Mensah ${ }^{3}$, Peter Paul Dapare ${ }^{1}$, \\ Eric Mishio Bawa ${ }^{1}$ \\ ${ }^{1}$ Department of Biomedical Laboratory Science, University for Development Studies, Tamale, Ghana \\ ${ }^{2}$ Department of Molecular Medicine, Kwame Nkrumah University of Science and Technology, Kumasi, Ghana \\ ${ }^{3}$ Department of Medical Laboratory Technology, Kwame Nkrumah University of Science and Technology, Kumasi, Ghana
}

Email address:

anafiu@uds.edu.gh (N. Amidu)

${ }^{*}$ Corresponding author

\section{To cite this article:}

Nafiu Amidu, Benedict Boateng Antuamwine, Martin Awe Akilla, William Kwame Boakye Ansah Owiredu, Otchere Addai-Mensah, Peter Paul Dapare, Eric Mishio Bawa. Leucocyte Differential Count and Pregnancy Induced Hypertension: Implication for Risk and Disease Assessment. American Journal of Laboratory Medicine. Vol. 5, No. 1, 2020, pp. 32-41. doi: 10.11648/j.ajlm.20200501.15

Received: January 8, 2020; Accepted: January 31, 2020; Published: February 14, 2020

\begin{abstract}
PIH accounts significantly for maternal and perinatal mortality, especially in rural communities. The sudden onset of PIH and the lack of definitive case management is worrisome. The study analysed differential leucocytes count on PIH outcomes. This is a case-control study of 40 PIH $(8 \mathrm{GH}, 16 \mathrm{PE}$ and $16 \mathrm{EC})$ and 60 normotensive women who visited the Bolgatanga Regional Hospital, Ghana. Data on socio-demographics, clinical history and laboratory evaluations on blood and placental tissues were collected and analysed. Lymphocytes count was markedly increased in PIH women, while total leucocytes, neutrophils and NLR were significantly decreased. Lymphocytes count increased with severity of PIH ( $p=0.0037)$. A correlation $(r=-0.9565, p<0.0001)$ between lymphocyte and neutrophil counts was observed among PIH subjects. Oxidative stress was more associated with PIH compared to controls. Stillbirths was associated with PIH mothers with high leucocytes count. NLR (AUC; 0.857, $\mathrm{p}<0.0001$ ) and lymphocytes count (AUC; 0.822, $\mathrm{p}<0.0001$ ) were optimal for prognosis of PIH. Wide variations in NLR and lymphocytes count during pregnancy necessitates urgent attention.
\end{abstract}

Keywords: Preeclampsia, Gestational Hypertension, Differential Leucocytes Count, Neutrophil-to-Lymphocyte Ratio, Risk Assessment, Stillbirth

\section{Introduction}

Maternal deaths caused by hypertensive disorders of pregnancy globally stand at over 60,000 cases per year, with pregnancy-induced hypertension (PIH) complicating $6-10 \%$ of all pregnancies. The statistics is higher in low- and middle-income countries, where about $99 \%$ of these maternal deaths occur $[1,2]$. PIH only develops as a consequence of pregnancy and regresses after delivery. It is defined as hypertension starting after 20 weeks of gestation with or without proteinuria including gestational hypertension $(\mathrm{GH})$, preeclampsia (PE) and eclampsia (EC) [3].
In Ghana, the maternal mortality ratio is 340 per 100,000 live births compared to 17 deaths per 100,000 live births in the United States [2, 4]. Around $32 \%$ of maternal deaths and perinatal deaths of 106 per 1000 births, occurring in Tertiary hospitals in Ghana are attributed to hypertensive disorders of pregnancy, with the majority of these mortalities linked to PIH [5-7]. These mortality rates are even more daunting in rural Ghana, where access to adequate perinatal care is lacking.

The pathophysiology of PIH is not fully understood, though inflammation has been established to play a role, promoting the upregulation of proinflammatory $\mathrm{CD} 4+\mathrm{T}$ cells in systemic circulation [8]. Diagnosis is often elusive 
due to variable clinical presentations, with patients even with the severe disease capable of remaining asymptomatic. This makes the condition complex to manage, almost entirely unattainable in deprived communities. Accurate prediction of women at increased risk of PIH however could lead to better antenatal care and a possible reduction in complications.

In recent years, it has been reported that individual components of differential leucocyte counts, remarkably neutrophil and lymphocyte counts together with their ratio; NLR (neutrophil-to-lymphocyte ratio) are characteristic markers of systemic inflammation and endothelial dysfunction, highlighting their usefulness in predicting/managing disease conditions such as $\mathrm{PIH}$, whose pathogenesis is promoted by inflammation and endothelial dysregulation [9-11]. Early assessment of preeclampsia and eclampsia especially in rural communities is necessary to prevent $\mathrm{PIH}$ complications and their associated increased maternal and perinatal mortalities.

Differential leucocyte count forms part of the complete blood cell count test which is readily available in almost all regional and district clinical laboratories and health facilities [12]. This grants clinicians and healthcare providers in rural Ghana timely access to the potential markers of PIH notably NLR. Despite the markers of oxidative stress such as malondialdehyde (MDA), total antioxidant capacity (TAC) and oxidative stress index (OSI) among others show high sensitivity in predicting $\mathrm{PIH}$, their routine measurement and accessibility, especially at remote areas is non-existent [13, 14]. In such situations the NLR will offer a huge advantage for the management of PIH.

This study therefore seeks to evaluate the prognostic value of differential leucocyte counts in the risk and disease assessment of PIH by a method that is rapid, cheap and widely available. Thus, differential leucocyte count at different stages of PIH was compared with normotensive controls. We showed increased lymphocyte counts and remarkably decreased NLR among a group of PIH mothers.

\section{Materials and Method}

\subsection{Study Design and Population}

This is a case-control study conducted among pregnant women visiting the Bolgatanga Regional Hospital, Ghana from September, 2015 through May, 2016. Forty women presenting with $\mathrm{PIH}(8 \mathrm{GH}, 16 \mathrm{PE}$ and $16 \mathrm{EC})$ and 60 normotensive pregnant women were recruited into the study. $\mathrm{GH}$ was defined as any new onset of hypertension (systolic blood pressure $\geq 140 \mathrm{mmHg}$ and/or diastolic blood pressure $\geq 90 \mathrm{mmHg}$ ) at $\geq 20$ weeks of gestation in the absence of proteinuria or new signs of end-organ dysfunction. PE was defined as pregnant women who were diagnosed with sustained diastolic blood pressure above $90 \mathrm{mmHg}$ and proteinuria up to $300 \mathrm{mg} / 24 \mathrm{hr}$ urinary sample in the absence of urinary tract infection. EC was defined as unclassified hypertension at $\geq 20$ weeks of gestation accompanied by seizures superimposed on the syndrome of pre-eclampsia. The classifications were based on the criteria outlined by the American College of Obstetricians and Gynecologists [15]. Pregnant women without any underlying pathological condition were classified as normotensive.

\subsection{Demographics}

Data on socio-demographic characteristics (age, gestational age, parity, gravidity) and clinical history were obtained from structured interviews and medical records respectively.

\subsection{Anthropometric Characteristics}

Adhering to WHO standard procedure, an electronic scale and a meter rule were used to measure respectively body weight and height as previously described [16]. BMI was calculated by dividing weight $(\mathrm{Kg})$ by height squared $\left(\mathrm{m}^{2}\right)$.

\subsection{Blood Pressure Measurement}

Using a mercury sphygmomanometer and a stethoscope, trained personnel measured the blood pressure of participants. Measurements were taken from the left upper arm after the subjects had been sitting for over 5 minutes and duplicate readings were documented at four-hour intervals.

\subsection{Laboratory Investigations}

\subsubsection{Complete Blood Cell Count}

After delivery, $3 \mathrm{ml}$ of venous blood was drawn from the antecubital vein of mothers and analysed within one hour for complete blood cell counts, including 5-part leucocyte differential count employing the $\mathrm{XN}-450$ haematology autoanalyser (Sysmex, Hamburg, Germany).

\subsubsection{Placental Tissue Homogenization}

About $25-60 \mathrm{~g}$ piece $(1-2$ cotyledons $)$ of placenta tissues were cut from the villous tree of each placentae within one hour after delivery and extensively rinsed with PBS to remove excess blood. This was either stored frozen at $-80^{\circ} \mathrm{C}$ or immediately homogenized in about $0.5 \mathrm{~g}$ tissue per $1 \mathrm{ml}$ of ice-cold PBS. The homogenate was centrifuged for $15 \mathrm{~min}$ at $5000 \mathrm{~g}$ at $-20^{\circ} \mathrm{C}$ after which the supernatant was harvested and assayed immediately or aliquoted and stored at $-80^{\circ} \mathrm{C}$.

\subsubsection{Placental Homogenate Malondialdehyde}

The determination of oxidative stress levels was analysed by the method described by Harma and colleagues [17]. $0.5 \mathrm{ml}$ of placenta homogenate was treated with $2.5 \mathrm{ml}$ of $20 \%$ trichloroacetic acid and then thoroughly mixed with $1.0 \mathrm{ml}$ of $0.67 \%$ thiobarbituric acid. The mixture was then incubated at $100^{\circ} \mathrm{C}$ for $30 \mathrm{~min}$. After cooling, the samples were extracted with $4.0 \mathrm{ml}$ of n-butanol, vortexed for $30 \mathrm{sec}$ and then centrifuged at $5000 \mathrm{~g}$ for $10 \mathrm{~min}$. The absorbance of the supernatant at $535 \mathrm{~nm}$ wavelength was measured employing the spectrophotometer (spec. City, Country) and expressed in $\mu \mathrm{mol} / \mathrm{L}$. 


\subsubsection{Placental Homogenate Total Antioxidant Capacity Test}

The total antioxidant capacity (TAC) was measured adopting the Koracevic et al., method [18]. It is based on the principle that a standardised solution of Fe-EDTA complex reacts with $\mathrm{H}_{2} \mathrm{O}_{2}$ to give hydroxyl radical, a reactive oxygen species. This reactive oxygen species degrades benzoate to produce thiobarbiturate reacting substances (TBARS). The antioxidant from the added sample causes suppression of the production of TBARS. Therefore, the decrease in the concentration of TBARS as a result of antioxidants is measured spectrophotometrically and serves as the concentration of antioxidants present in the placenta sample added.

\subsubsection{Total Peroxide Concentration}

The total peroxide concentration (TP) was determined using the FOX2 method [17]. The FOX2 test system is based on oxidation of ferrous ion to ferric ion by various types of peroxides contained within the placenta samples, to produce a coloured ferric-xylenol orange complex whose absorbency can be measured at $560 \mathrm{~nm}$.

\subsubsection{Oxidative Stress Index}

The oxidative stress index (OSI) is the percentage ratio of the TP to the TAC and indicates the extent of oxidative stress [17]. The OSI was calculated as;

$$
\text { OSI }=\frac{\mathrm{TP}, \mu \mathrm{mol} \mathrm{L}^{-1}}{\mathrm{TAC}, \mu \mathrm{mol} \text { Trolox equivalent } \mathrm{L}^{-1}} \times 100
$$

\subsection{Statistical Analysis}

Data analyses were performed using GraphPad Prism version 6. Continuous variables were presented as means and standard deviations while categorical variables were presented as frequencies and percentages. Unpaired student ttest was used to compare mean differences among continuous variables while Fisher's exact test was used to compare mean differences among categorical variables. Receiver operating characteristic curve (ROC) was employed to test for the differential leucocyte count that could offer better assessment of PIH. Pearson coefficient was employed to test for correlation between differential leucocyte counts and oxidative stress markers. Statistical significance was set at $\mathrm{p}<0.05$.

\subsection{Ethical Approval}

The study was approved by the Navrongo Health Research Centre Institutional Review Board. Written informed consent was obtained from all participants.

\section{Results}

One hundred pregnant women participated in the study, out of which 40 were diagnosed of PIH while the rest were normotensive. Both categories of participants were identical by age and the other demographic characteristics, except gestational age which was shortened among PIH mothers.
Clinical characteristics however were widely varied, as blood pressure and body mass index (BMI) were expectedly raised markedly in PIH mothers compared to normotensive mothers (Table 1).

Table 1. Demographic and clinical characteristics of Ghanaian PIH mothers.

\begin{tabular}{llll}
\hline Variables & PIH (40) & Control (60) & P-value \\
\hline Age (yrs.) & $29.4 \pm 6.6$ & $26.8 \pm 6.4$ & 0.1714 \\
Parity & $2.2 \pm 1.8$ & $1.3 \pm 1.5$ & 0.0814 \\
Gravidity & $3.1 \pm 1.8$ & $2.3 \pm 1.4$ & 0.1115 \\
Gestational age (wks.) & $37.3 \pm 3.2$ & $39.0 \pm 1.6$ & 0.0192 \\
SBP (mmHg) & $159.6 \pm 18.15$ & $111.7 \pm 10.32$ & $<0.0001$ \\
DBP (mmHg) & $101.4 \pm 11.62$ & $68.10 \pm 8.93$ & $<0.0001$ \\
BMI & $28.6 \pm 6.7$ & $25.0 \pm 4.5$ & 0.0274 \\
Leucocyte differential count (cells x 10 $/ \mathrm{L})$ & & \\
WBC & $10.36 \pm 3.60$ & $15.41 \pm 6.03$ & 0.0020 \\
NEU & $7.37 \pm 1.08$ & $8.21 \pm 0.95$ & 0.0053 \\
LYM & $1.88 \pm 0.95$ & $0.98 \pm 0.57$ & 0.0002 \\
MON & $0.63 \pm 0.24$ & $0.64 \pm 0.28$ & 0.8928 \\
EOS & $0.024 \pm 0.015$ & $0.017 \pm 0.015$ & 0.095 \\
BASO & $0.08 \pm 0.05$ & $0.06 \pm 0.08$ & 0.3475 \\
NLR & $4.84 \pm 2.37$ & $12.76 \pm 7.48$ & $<0.0001$ \\
$<4.0$ & $20(50.0 \%)$ & $6(10.0 \%)$ & \\
$4.0-8.0$ & $14(35.0 \%)$ & $12(20.0 \%)$ & \\
$>8.0$ & $6(15.0 \%)$ & $42(70.0 \%)$ & $<0.0001$ \\
\hline
\end{tabular}

Continuous data are presented as mean $\pm \mathrm{SD}$ and categorical variable as number with percentage in parenthesis. PIH; pregnancy induced hypertension, SBP; systolic blood pressure, DBP; diastolic blood pressure, BMI; body mass index, WBC; white blood cell count, NEU; neutrophils, LYM; lymphocytes, MON; monocytes, EOS; eosinophils, BASO; basophils, NLR; neutrophil-to-lymphocyte ratio

Additionally, leucocyte differential counts, notably total white blood cells (WBC, $\mathrm{p}=0.002$ ) and neutrophils (NEU, $\mathrm{p}=0.0053$ ) were decreased in PIH mothers while their lymphocytes (LYM, p=0.0002) were increased. Their estimated NLR $(\mathrm{p}<0.0001)$ was also decreased as shown in Table 1 .

In order to investigate whether differential leucocyte counts could offer predictive assessment of the progression of PIH, leucocyte subpopulations were analysed in the different progressive stages of PIH. Out of the 40 pregnant women diagnosed with $\mathrm{PIH}, 20 \%$ were diagnosed with gestational hypertension, while the remaining $80 \%$ were equally distributed between preeclampsia and eclampsia. Lymphocyte counts was observed to increase with increasing severity of PIH, with the highest numbers detected in women with the most severe stage of the condition, eclampsia who also demonstrated significant decrease in neutrophils (Figure 1). Significant decreases in NLR corresponded with worsening stages of PIH, with EC mothers showing the lowest estimates of NLR. The entire leucocyte population was moderately decreased in GH and EC subjects as shown in Figure 1. No significant changes in the numbers of monocytes, eosinophils and basophils were observed as PIH progressed. 


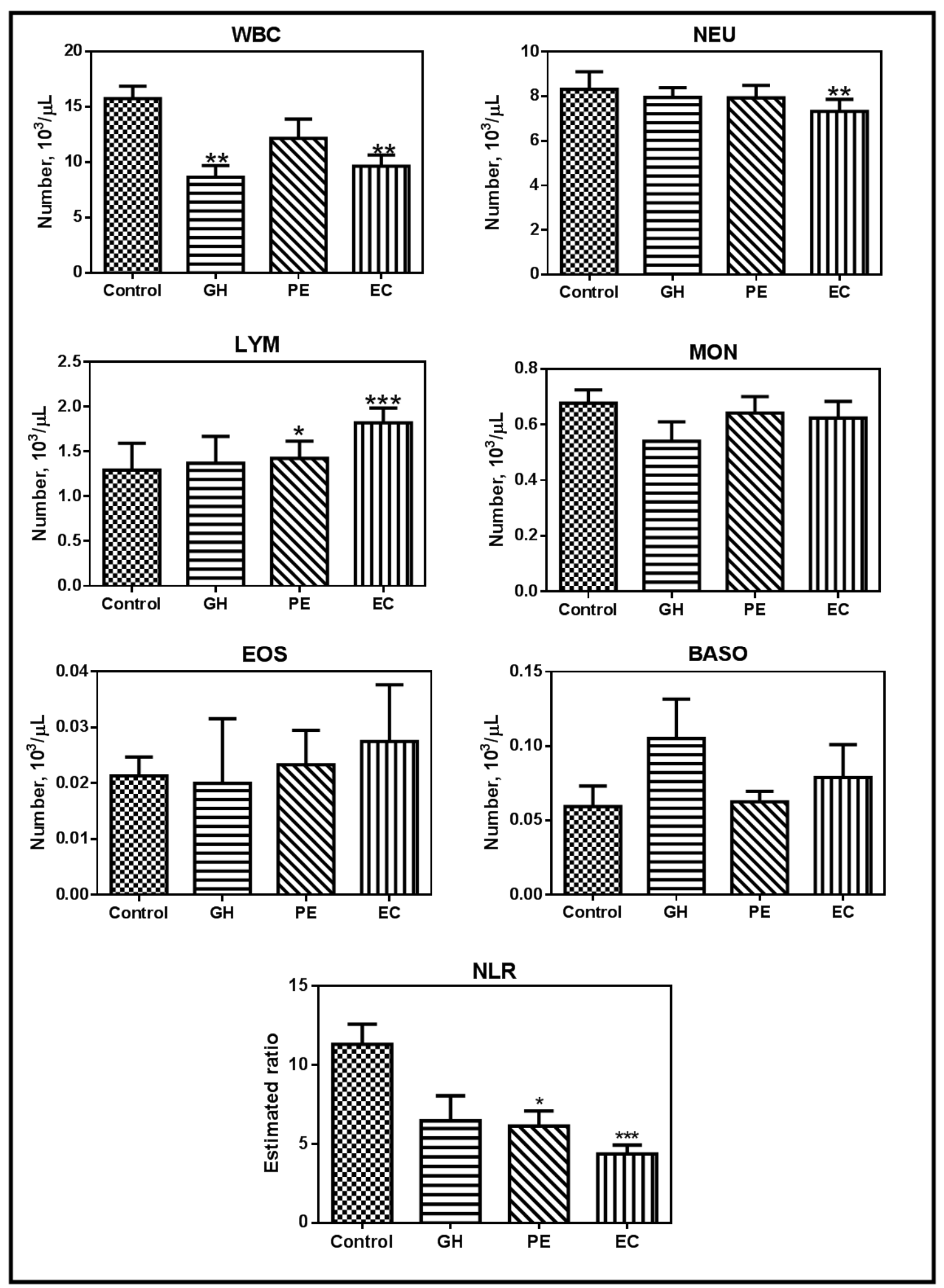

Figure 1. Leucocyte differential counts in various stages of PIH. PIH; pregnancy induced hypertension, NLR; neutrophil-to-lymphocyte ratio, GH; gestational hypertension, PE; preeclampsia, EC; eclampsia. Values are mean \pm SEM and significantly different from control using unpaired student $t$-test: *; $p<0.05$, **; $p<0.01, * * * ; p<0.001$.

When placental sections of the study population were homogenized and analysed for markers of oxidative stress, it was observed that malondialdehyde and oxidative stress index were markedly increased in the hypertensive mothers compared to their normotensive counterparts. It was further observed that the placentas of PIH mothers were heavily stressed, demonstrating marked and moderate reduction in total antioxidant capacity and catalase activity respectively (Figure 2). 


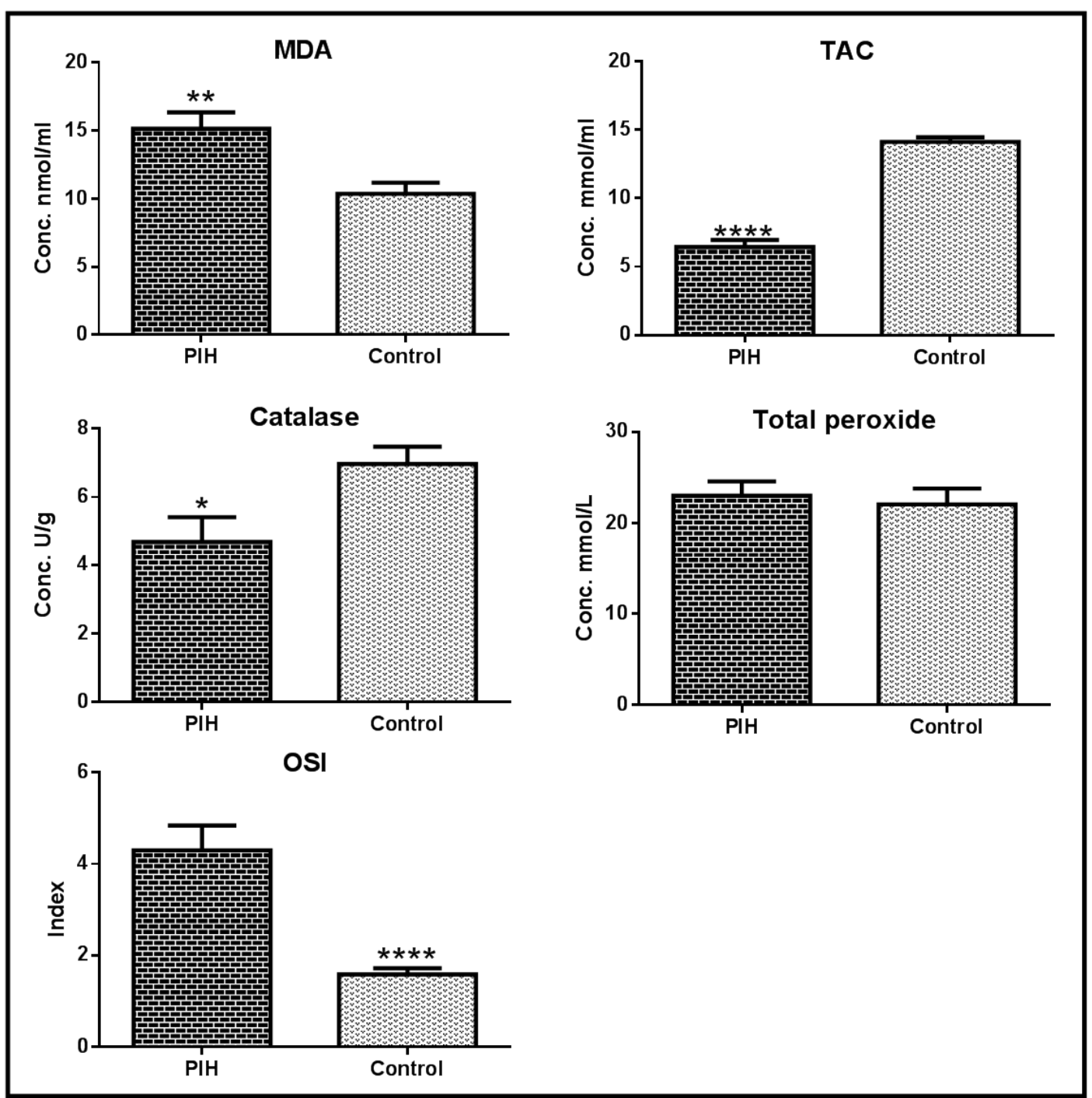

Figure 2. The concentration levels of oxidative stress markers between PIH and control subjects. PIH; pregnancy induced hypertension, MDA; malondialdehyde, TAC; total antioxidant capacity, OSI; oxidative stress index. Values are mean \pm SEM and significantly different from control using unpaired student t-test: *; $p<0.05,{ }^{* *} ; p<0.01,{ }^{* * *} ; p<0.001,{ }^{* * * * ;} p<0.0001$

Despite oxidative stress markers and leucocyte differential populations showing remarkable variations in PIH subjects compared to their controls, there was however no significant correlation between leucocyte differential counts and oxidative stress markers as shown in Table 2. A significant negative correlation between lymphocyte and neutrophil counts $(\mathrm{r}=-0.96,<0.0001)$ was however observed in $\mathrm{PIH}$ subjects (Table 2).

Table 2. Pearson Correlation Co-efficient between leucocyte differential populations and oxidative stress markers in PIH mothers.

\begin{tabular}{|c|c|c|c|c|c|c|c|c|c|c|c|c|}
\hline & WBC & NEU & LYM & MON & EOS & BASO & NLR & MDA & TAC & Catalase & T. Per & OSI \\
\hline WBC & & -0.11 & 0.29 & -0.39 & -0.32 & -0.26 & -0.23 & 0.17 & 0.02 & 0.11 & 0.13 & 0.02 \\
\hline NEU & -0.11 & & $-0.96 * * * *$ & -0.34 & -0.43 & -0.31 & $0.77 * * *$ & -0.24 & 0.33 & -0.00 & -0.13 & -0.36 \\
\hline LYM & 0.29 & $-0.96 * * * *$ & & 0.10 & 0.26 & 0.22 & $-0.79 * * *$ & 0.23 & -0.27 & 0.02 & 0.11 & 0.31 \\
\hline MON & -0.39 & -0.34 & 0.10 & & 0.21 & 0.03 & -0.15 & -0.05 & -0.24 & -0.17 & 0.17 & 0.30 \\
\hline EOS & -0.32 & -0.43 & 0.26 & 0.21 & & $0.55^{*}$ & -0.21 & 0.33 & -0.13 & 0.24 & 0.048 & 0.09 \\
\hline BASO & -0.26 & -0.31 & 0.22 & 0.03 & $0.55^{*}$ & & -0.22 & 0.13 & -0.36 & -0.09 & -0.19 & 0.13 \\
\hline NLR & -0.23 & $0.77 * * *$ & $-0.79 * * *$ & -0.15 & -0.21 & -0.22 & & -0.15 & 0.40 & 0.30 & -0.17 & -0.43 \\
\hline TAC & 0.02 & 0.33 & -0.27 & -0.24 & -0.13 & -0.36 & 0.40 & 0.38 & & $0.56^{*}$ & 0.12 & $-0.89 * * * *$ \\
\hline Catalase & 0.11 & 0.00 & 0.02 & -0.17 & 0.24 & -0.09 & 0.30 & $0.51 *$ & $0.56^{*}$ & & 0.37 & -0.42 \\
\hline T. Per & 0.13 & -0.13 & 0.11 & 0.17 & 0.05 & -0.19 & -0.17 & $0.51 *$ & 0.12 & 0.37 & & 0.21 \\
\hline OSI & 0.02 & -0.36 & 0.31 & 0.30 & 0.09 & 0.13 & -0.40 & -0.34 & $-0.89 * * * *$ & -0.42 & 0.21 & \\
\hline
\end{tabular}

*Correlation is significant at the 0.05 level (2-tailed), **. Correlation is significant at the 0.01 level (2-tailed), ***. Correlation is significant at the 0.001 level (2-tailed). WBC; white blood cell counts, NEU; neutrophils, LYM; lymphocytes, MON; monocytes, EOS; eosinophils, BASO; basophils, NLR; neutrophil-tolymphocyte ratio; MDA; malondialdehyde, TAC; total antioxidant capacity, T. Per; total peroxide, OSI; oxidative stress index. 
An assessment of differential leucocyte counts on the delivery and foetal outcome of PIH mothers revealed that total leucocyte count was highly elevated in mothers who had still births as shown in Table 3. These findings supported investigations on the use of differential leucocytes counts as prognostic and assessment tools for $\mathrm{PIH}$.

Table 3. Stratification of PIH mothers based on differential leucocyte counts and pregnancy outcome.

\begin{tabular}{lllll}
\hline \multirow{2}{*}{ Parameter } & Delivery outcome & & Foetal outcome & Still birth \\
\cline { 2 - 5 } & Term & Preterm & Live birth & $26.19 \pm 5.50^{* * * * *}$ \\
\hline WBC & $10.20 \pm 2.41$ & $10.71 \pm 5.69$ & $9.97 \pm 2.31$ & $7.24 \pm 1.61$ \\
NEU & $7.38 \pm 1.11$ & $7.34 \pm 1.12$ & $7.39 \pm 0.98$ & $1.05 \pm 0.45$ \\
LYM & $1.79 \pm 0.83$ & $1.85 \pm 1.25$ & $1.75 \pm 0.74$ & $0.41 \pm 0.21$ \\
MON & $0.64 \pm 0.25$ & $0.61 \pm 0.25$ & $0.67 \pm 0.23$ & $0.04 \pm 0.03$ \\
EOS & $0.02 \pm 0.01$ & $0.03 \pm 0.02$ & $0.06 \pm 0.05$ & $0.07 \pm 0.01$ \\
BASO & $0.07 \pm 0.04$ & $0.09 \pm 0.06$ & $0.07 \pm 0.04$ & $3.34 \pm 2.52$ \\
NLR & $5.11 \pm 2.58$ & $4.49 \pm 2.28$ & $5.10 \pm 2.37$ & \\
\hline
\end{tabular}

WBC; White blood cells, NEU; neutrophils, LYM; lymphocytes, MON; monocytes, EOS; eosinophils, BASO; basophils, NLR; neutrophil-to-lymphocyte ratio. Values are mean $\pm \mathrm{SD}$. Comparisons between two groups was done using unpaired student t-test: $* * * * ; \mathrm{p}<0.0001$.

ROC curve analysis showed NLR to be best at predicting $\mathrm{PIH}$ disease (AUC; 0.857, $\mathrm{p}<0.0001$ ) followed by lymphocyte count (AUC; 0.822, p <0.0001) and then neutrophil count (AUC; 0.756, $\mathrm{p}=0.0001$ ) compared to the other white cell differential counts (Figure 3).

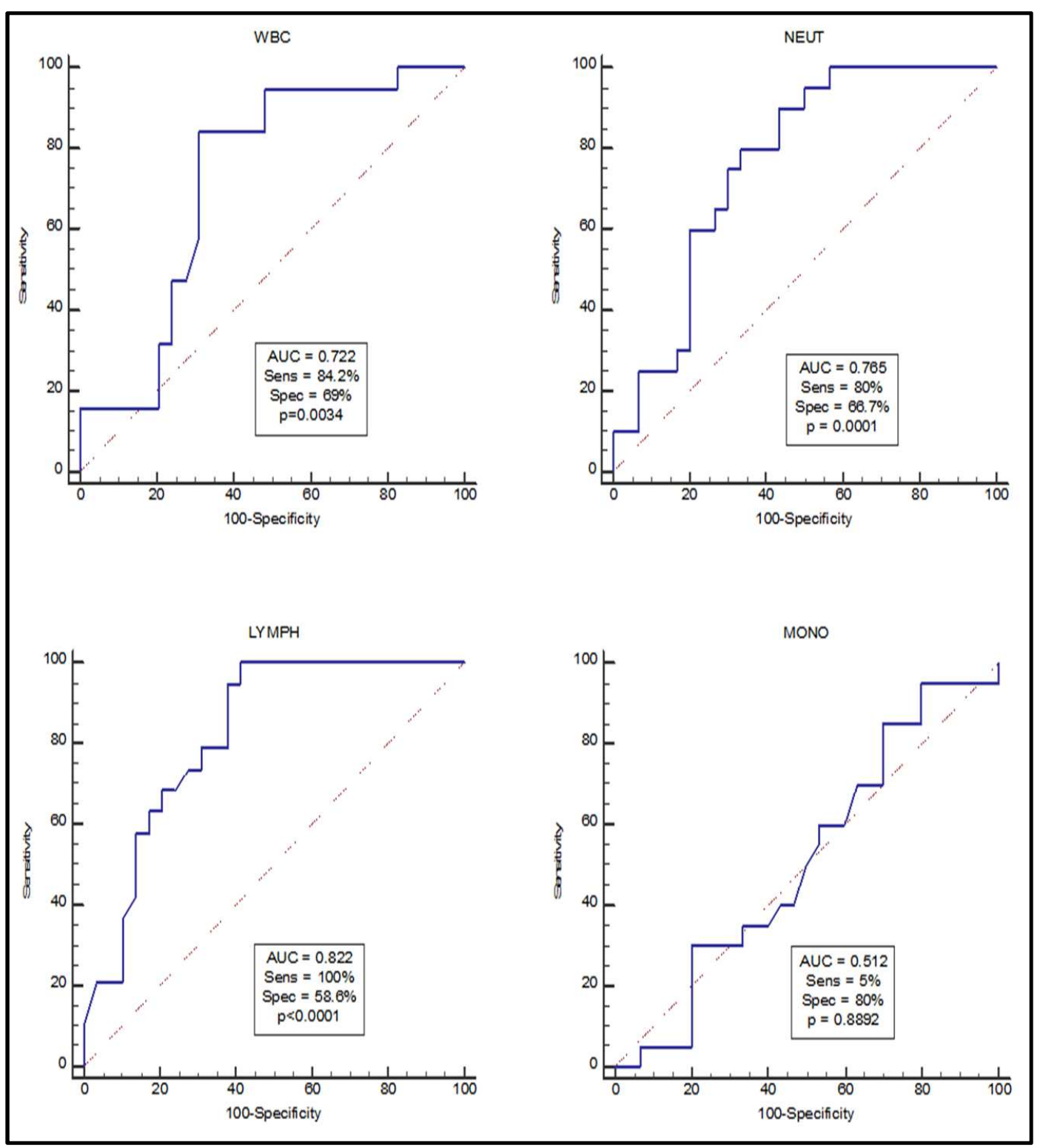




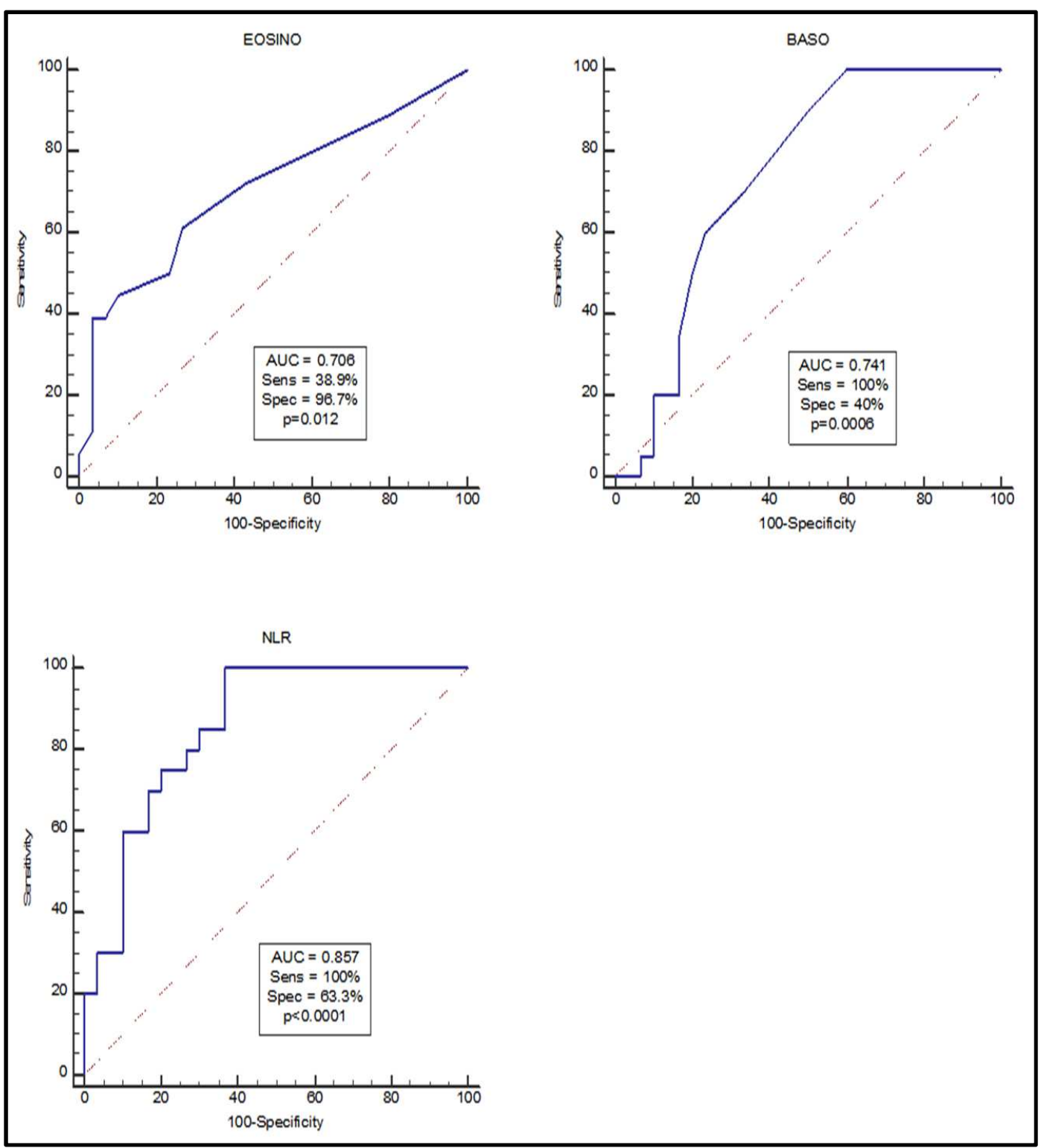

Figure 3. ROC curve analysis of differential leucocyte counts in PIH disease prediction. WBC; White blood cells, NEU; neutrophils, LYM; lymphocytes, MON; monocytes, EOS; eosinophils, BASO; basophils, NLR; neutrophil-to-lymphocyte ratio, AUC; area under the curve, Sens; sensitivity, Spec; specificity.

\section{Discussion}

$\mathrm{PIH}$ as a leading cause of maternal and perinatal mortality and morbidity even poses harsher threats to women in rural communities due to their inadequate access to quality healthcare delivery. The present study demonstrates significantly decreased total WBC and neutrophil counts among PIH women while their lymphocyte counts were significantly increased compared to normotensive pregnant women. A determination of NLR was found to be markedly decreased among PIH subjects compared to their controlled counterparts. Despite poor understanding on the pathophysiology of PIH, inflammation is well established to play a role in the pathogenesis of the condition, upregulating the production of proinflammatory CD4+ T-cells $[8,19]$. Our findings of marked elevation of lymphocytes in systemic circulation of PIH mothers could be suggestive of an upsurge of CD4+ T-cell population. Additionally, the decreased neutrophil count and consequently decreased total WBC count in PIH subjects could be hypothesised to be a resulting effect of the increased production of lymphocytes, which may affect neutrophil production and their distribution in systemic circulation.

Increased neutrophil numbers have been suggested to account for leucocytosis in PIH by several studies [9, 20-22] and thus mechanisms that trigger neutrophil decline eventually result in a decrease in the total WBC count as observed in our study. Several studies [8, 19, 23, 24] have identified immune imbalance, characterized by increases in proinflammatory CD4+ T-cells and cytokines along with decreases in regulatory $\mathrm{T}$-cells and anti-inflammatory cytokines, as a key mechanism in the pathophysiology of PIH. This may have accounted for the reduced neutrophil count among PIH subjects and also contributed to the 
reduced neutrophil to lymphocyte ratio (NLR) observed among PIH mothers. However, no differences in monocyte, eosinophil and basophil counts between PIH and normotensive mothers were observed.

The study population was fairly distributed by age, parity and gravidity as there were no significant differences between $\mathrm{PIH}$ and normotensive mothers. However, PIH mothers had a gestational age to be about 2 weeks younger than their normotensive mothers. This was not surprising as women diagnosed with PIH often do not carry the pregnancy to term by undergoing caesarean section as a case management approach $[25,26]$. Higher systolic and diastolic blood pressures in PIH mothers compared to controls confirmed hypertension in the study subjects. Additionally, the higher BMI observed among PIH mothers could have been triggered by underlying cardiovascular diseases such as obesity among others, that have been shown to have a strong association with PIH $[27,28]$.

In order to understand whether leucocyte numbers in systemic circulation could be useful in the assessment of the severity of PIH, we evaluated leucocyte differential counts and NLR among different clinical stages of PIH; from the mild GH through PE, a moderate form of the condition and then EC, the worst stage of the disease. Previous studies have shown the usefulness of leucocyte differential counts in the assessment and management of several disease conditions including cardiovascular disease and related complications $[29,30]$, ulcerative colitis [31] and cancers [11, 32] among others. In the present study, significant variations were observed in leucocyte differential counts among subjects presenting with different clinical stages of PIH. A moderate significant rise in lymphocyte counts were noted in PIH mothers diagnosed of PE while those with EC demonstrated a marked increase in lymphocytes count. Those with GH on the other hand did not show any significant increase in lymphocyte counts. This phenomenon of lymphocyte expansion correlating with severity of PIH demonstrates the involvement of lymphocytes in the pathogenesis and progression of PIH. Recent studies have established PE to be associated with a dysregulation of cytokines which largely contributes to the elevation of lymphocyte populations [33, 34]. Extended dysregulation as may be seen in EC will further elevate the lymphocyte population as shown in our study and other studies [33, 35, 36]. Significant decrease in WBC and neutrophils were respectively observed among mothers with GH and EC while NLR was observed to decrease with increasing severity of PIH, with EC mothers recording the smallest NLR.

Our findings of decreasing NLR with increasing severity of PIH is quite contrary to several studies that have reported high NLR among PIH subjects [9, 10, 37]. NLR which is influenced by neutrophil and lymphocyte populations, is affected greatly when one of the populations increases or decreases in relation to the other. Neutrophil count is unexpectedly decreased in PIH subjects compared to controls. The marked elevation of lymphocytes observed in the present study may have compensated for the significant decrease in neutrophil counts. Furthermore, elevated NLR has been shown to correlate with lymphocytopenia in patients, resulting in a suboptimal lymphocyte mediated immune response to disease $[38,39]$. The vice versa could be established, where a decline in NLR correlates with lymphocytosis as observed in our study. To our knowledge, this is the first study to establish decreasing NLR with progression of PIH, showing that peripheral lymphocyte count was most significantly related to PIH severity. Chiyao Hsueh and colleagues [40] in their study demonstrated that lower neutrophil counts, NLR and elevated lymphocytes correlated with laryngeal squamous cell carcinoma progression.

Peripheral lymphocyte and neutrophil counts together with NLR proved to be better assessment tools for PIH compared to other leucocyte parameters. Based on ROC cut off criteria, the predictability of PIH by each of the white cell differential counts was assessed. The NLR was found to offer the best prediction of PIH, followed by peripheral lymphocytes count and then neutrophil counts. NLR is increasingly gaining popularity as a sensitive marker for diagnosis or assessment of diseases [37, 41, 42]. Its significance in PIH cannot be overemphasized as abnormal changes in immune-mediated inflammation play a central role in the pathogenesis of preeclampsia. It was interesting to note also the high $\mathrm{PIH}$ predictability score for lymphocytes and neutrophils, suggestive of sensitive markers for the management of PIH. Despite the availability of complete blood cell counts as a routine test in several regional and district clinical laboratory settings [12], the management of PIH especially in rural areas is poor. Inadequate utilization of full blood cell count results by healthcare providers may be a hinderance to improved management of PIH. Our findings show that when leucocyte differential counts of patients are closely observed, they could offer preliminary diagnosis or an assessment of PIH, a step that would be priceless in remote communities where PIH contributes greatly to maternal and infant mortality [43, 44].

The pathogenesis of PIH is facilitated by oxidative stress within the placenta $[13,14,45]$. To study whether oxidative stress had impact on leucocyte differential counts, we first analysed markers of oxidative stress in the placentas of PIH and normotensive mothers. Assayed levels of MDA and OSI were significantly higher in the placenta of PIH subjects compared to those of normotensive subjects. This supports the findings that PIH is characterised by on-going cellular activities in the placenta that results in the generation of reactive oxidative species (ROS) [13, 14]. Additionally, assayed levels of TAC and catalase were significantly reduced in the placenta of PIH mothers, thus accounting for the high OSI. Despite significant variations in the markers of oxidative stress, correlations with leucocyte differential counts were not significant. This suggests that each of the oxidative stress markers has no direct impact on WBC differentials. To our prediction and per the findings of other studies [17, 46, 47], a significant negative correlation between TAC and OSI was observed while that of catalase 
was positive. Additionally, positive correlation between MDA and catalase was significant, further distinguishing between the two study populations.

The present study also reports on the association between leucocyte differential counts and neonatal outcomes. Aside WBC counts which was significantly increased among PIH mothers with still births, there were no significant variations between leucocyte differential levels and delivery outcomes in PIH mothers. High WBC counts have been reported to be associated with stillbirths [48-50], yet the exact mechanism is unknown. The absence of significant variations in leucocyte differential counts between stillbirth and normal birth PIH mothers may suggest that other inflammatory cells and or proteins could be triggers of foetal demise. Further studies are therefore required to provide an in-depth understanding on the underlying mechanisms.

\section{Conclusion}

In this study, we report on an unexpected decrease of neutrophil-to-lymphocyte ratio in $\mathrm{PIH}$, shedding more light on the role of lymphocytes and neutrophil in $\mathrm{PIH}$ pathogenesis and expanding the scope of research in the field. In view of the poor management of PIH, peripheral blood count of neutrophils, lymphocytes and NLR which showed to be sensitive tools for PIH disease diagnosis and or assessment would be essential for the management of the condition especially in deprived communities.

\section{Funding}

The study was financed by the authors

\section{Competing Interests}

Authors declare that no competing interests exists

\section{References}

[1] Duhig, K., B. Vandermolen, and A. Shennan, Recent advances in the diagnosis and management of pre-eclampsia. F1000 Research, 2018. 7.

[2] Organization, W. H. and Unicef, Trends in maternal mortality: 1990 to 2013: estimates by WHO, UNICEF, UNFPA, The World Bank and the United Nations Population Division. 2014.

[3] Ananth, C. V. and O. Basso, Impact of pregnancy-induced hypertension on stillbirth and neonatal mortality in first and higher order births: a population-based study. Epidemiology (Cambridge, Mass.), 2010. 21 (1): p. 118.

[4] Creanga, A. A., et al., Pregnancy-related mortality in the United States, 2011-2013. Obstetrics and gynecology, 2017. 130 (2): p. 366.

[5] Adu-Bonsaffoh, K., et al., Perinatal outcomes of hypertensive disorders in pregnancy at a tertiary hospital in Ghana. BMC pregnancy and childbirth, 2017. 17 (1): p. 388.
[6] Lee, Q. Y., et al., Maternal mortality in Ghana: a hospitalbased review. Acta obstetricia et gynecologica Scandinavica, 2012. 91 (1): p. 87-92.

[7] Adu-Bonsaffoh, K., et al., Maternal deaths attributable to hypertensive disorders in a tertiary hospital in Ghana. International Journal of Gynecology \& Obstetrics, 2013. 123 (2): p. 110-113.

[8] Harmon, A. C., et al., The role of inflammation in the pathology of preeclampsia. Clinical science, 2016. 130 (6): p. 409-419.

[9] Serin, S., et al., Is neutrophil/lymphocyte ratio a useful marker to predict the severity of pre-eclampsia? Pregnancy Hypertension: An International Journal of Women's Cardiovascular Health, 2016. 6 (1): p. 22-25.

[10] Gogoi, P., et al., Neutrophil-to-lymphocyte ratio and platelet indices in pre-eclampsia. International Journal of Gynecology \& Obstetrics, 2019. 144 (1): p. 16-20.

[11] Szkandera, J., et al., External validation of the derived neutrophil to lymphocyte ratio as a prognostic marker on a large cohort of pancreatic cancer patients. PloS one, 2013. 8 (11): p. e78225.

[12] Briggs, C., et al., ICSH Guideline for worldwide point-of-care testing in haematology with special reference to the complete blood count. International journal of laboratory hematology, 2008. 30 (2): p. 105-116.

[13] Aouache, R., et al., Oxidative stress in preeclampsia and placental diseases. International journal of molecular sciences, 2018. 19 (5): p. 1496.

[14] Matsubara, K., et al., Nitric oxide and reactive oxygen species in the pathogenesis of preeclampsia. International journal of molecular sciences, 2015. 16 (3): p. 4600-4614.

[15] Obstetricians, A. C. o. and Gynecologists, Hypertension in pregnancy. Report of the American College of Obstetricians and Gynecologists' task force on hypertension in pregnancy. Obstetrics and gynecology, 2013. 122 (5): p. 1122.

[16] Amidu, N., et al., Use of Selected Anthropometric Indices for Screening Hypertension in an Adult Ghanaian Population. Asian Journal of Medical Principles and Clinical Practice, 2018: p. 1-9.

[17] Harma, M., M. Harma, and O. Erel, Measurement of the total antioxidant response in preeclampsia with a novel automated method. European Journal of Obstetrics \& Gynecology and Reproductive Biology, 2005. 118 (1): p. 47-51.

[18] Koracevic, D., et al., Method for the measurement of antioxidant activity in human fluids. Journal of clinical pathology, 2001. 54 (5): p. 356-361.

[19] LaMarca, B., et al., Identifying immune mechanisms mediating the hypertension during preeclampsia. American Journal of Physiology-Regulatory, Integrative and Comparative Physiology, 2016. 311 (1): p. R1-R9.

[20] Canzoneri, B. J., et al., Increased neutrophil numbers account for leukocytosis in women with preeclampsia. American journal of perinatology, 2009. 26 (10): p. 729-732.

[21] Kurtoglu, E., et al., May ratio of neutrophil to lymphocyte be useful in predicting the risk of developing preeclampsia? A pilot study. The Journal of Maternal-Fetal \& Neonatal Medicine, 2015. 28 (1): p. 97-99. 
[22] Mannaerts, D., et al., Are neutrophil/lymphocyte ratio (NLR), platelet/lymphocyte ratio (PLR), and/or mean platelet volume $(M P V)$ clinically useful as predictive parameters for preeclampsia? The Journal of Maternal-Fetal \& Neonatal Medicine, 2019. 32 (9): p. 1412-1419.

[23] Cornelius, D. C., Preeclampsia: from inflammation to immunoregulation. Clinical Medicine Insights: Blood Disorders, 2018. 11: p. 1179545X17752325.

[24] Vargas-Rojas, M I., H. Solleiro-Villavicencio, and E. SotoVega, Th1, Th2, Th17 and Treg levels in umbilical cord blood in preeclampsia. The Journal of Maternal-Fetal \& Neonatal Medicine, 2016. 29 (10): p. 1642-1645.

[25] Shibata, T., et al., Management of severe pregnancy-induced hypertension after 34 weeks of gestation: A prospective study to reduce the rate of cesarean section. Hypertension in pregnancy, 2016. 35 (1): p. 82-90.

[26] Amaral, L. M., et al., Pathophysiology and current clinical management of preeclampsia. Current hypertension reports, 2017. 19 (8): p. 61

[27] Owiredu, W., et al., Putative risk factors of pregnancy-induced hypertension among Ghanaian pregnant women. Journal of Medical and Biomedical Sciences, 2012. 1 (3): p. 62-76.

[28] Somani, S., et al., To determine association of lipid profile, serum uric acid and body mass index as a marker for preeclampsia. Int J Cur Res Rev, 2015.7 (11): p. 53.

[29] Welsh, C., et al., Association of Total and Differential Leukocyte Counts with Cardiovascular Disease and mortality in the UK biobank. Arteriosclerosis, Thrombosis, and Vascular Biology, 2018. 38 (6): p. 1415-1423.

[30] Yoshimura, A., et al., Association of peripheral total and differential leukocyte counts with obesity-related complications in young adults. Obesity facts, 2015. 8 (1): p. 116.

[31] Torun, S., et al., Assessment of neutrophil-lymphocyte ratio in ulcerative colitis: a promising marker in predicting disease severity. Clinics and research in hepatology and gastroenterology, 2012. 36 (5): p. 491-497.

[32] Bahig, H., et al., Neutrophil count is associated with survival in localized prostate cancer. BMC cancer, 2015. 15 (1): p. 594.

[33] Taylor, E. B. and J. M. Sasser, Natural killer cells and T lymphocytes in pregnancy and pre-eclampsia. 2017, Portland Press Limited.

[34] Saito, S., et al., Th1/Th2/Th17 and regulatory T-cell paradigm in pregnancy. American journal of reproductive immunology, 2010. 63 (6): p. 601-610.

[35] Saito, S., et al., Quantitative analysis of peripheral blood Th0, Th1, Th2 and the Th1: Th2 cell ratio during normal human pregnancy and preeclampsia. Clinical and experimental immunology, 1999. 117 (3): p. 550.

[36] Borzychowski, A. M., et al., Changes in systemic type 1 and type 2 immunity in normal pregnancy and pre-eclampsia may be mediated by natural killer cells. European journal of immunology, 2005. 35 (10): p. 3054-3063.

[37] Wang, J., et al., Assessment efficacy of neutrophil-lymphocyte ratio and monocyte-lymphocyte ratio in preeclampsia. Journal of reproductive immunology, 2019.

[38] Fogar, P., et al., Decreased total lymphocyte counts in pancreatic cancer: an index of adverse outcome. Pancreas, 2006. 32 (1): p. 22-28.

[39] An, X., et al., Elevated neutrophil to lymphocyte ratio predicts survival in advanced pancreatic cancer. Biomarkers, 2010. 15 (6): p. 516-522.

[40] Hsueh, C., et al., The prognostic value of preoperative neutrophils, platelets, lymphocytes, monocytes and calculated ratios in patients with laryngeal squamous cell cancer. Oncotarget, 2017.8 (36): p. 60514.

[41] Seng, J. J. B., et al., Role of neutrophil to lymphocyte ratio (NLR), platelet to lymphocyte ratio (PLR) and mean platelet volume $(M P V)$ in assessing disease control in Asian patients with axial spondyloarthritis. Biomarkers, 2018. 23 (4): p. 335338 .

[42] Qin, B., et al., Neutrophil to lymphocyte ratio (NLR) and platelet to lymphocyte ratio (PLR) were useful markers in assessment of inflammatory response and disease activity in SLE patients. Modern rheumatology, 2016. 26 (3): p. 372-376.

[43] Ghulmiyyah, L. and B. Sibai. Maternal mortality from preeclampsialeclampsia. in Seminars in perinatology. 2012. Elsevier.

[44] Kande, A., et al., A study of maternal and foetal outcome in severe pre-eclampsia and eclampsia. IJAR, 2016. 2 (9): p. 490-492.

[45] Madazli, R., et al., The plasma and placental levels of malondialdehyde, glutathione and superoxide dismutase in pre-eclampsia. Journal of Obstetrics and Gynaecology, 2002. 22 (5): p. 477-480.

[46] Can, M., et al., Oxidative stress and apoptosis in preeclampsia. Tissue and Cell, 2014. 46 (6): p. 477-481.

[47] Kaur, G., et al., Alterations in lipid peroxidation and antioxidant status in pregnancy with preeclampsia. Molecular and cellular biochemistry, 2008. 313 (1-2): p. 37-44.

[48] Harrison, M. S., et al., Stillbirth, Inflammatory Markers, and Obesity: Results from the Stillbirth Collaborative Research Network. American journal of perinatology, 2018. 35 (11): p. 1071-1078.

[49] Yamauchi, A., et al., Causes of stillbirth: an analysis of 77 cases. Journal of Obstetrics and Gynaecology Research, 1999. 25 (6): p. 419-424.

[50] Blackwell, S., et al., Maternal and fetal inflammatory responses in unexplained fetal death. The Journal of MaternalFetal \& Neonatal Medicine, 2003. 14 (3): p. 151-157. 